Meta

Journal des traducteurs

Translators' Journal

\title{
Relations complexes
}

\section{Robert Dubuc}

Volume 20, numéro 3, septembre 1975

URI : https://id.erudit.org/iderudit/002635ar

DOI : https://doi.org/10.7202/002635ar

Aller au sommaire du numéro

Éditeur(s)

Les Presses de l'Université de Montréal

ISSN

0026-0452 (imprimé)

1492-1421 (numérique)

Découvrir la revue

Citer cet article

Dubuc, R. (1975). Relations complexes. Meta, 20(3), 206-208.

https://doi.org/10.7202/002635ar

Ce document est protégé par la loi sur le droit d'auteur. L'utilisation des services d'Érudit (y compris la reproduction) est assujettie à sa politique d'utilisation que vous pouvez consulter en ligne.

https://apropos.erudit.org/fr/usagers/politique-dutilisation/
Cet article est diffusé et préservé par Érudit.

Érudit est un consortium interuniversitaire sans but lucratif composé de l’Université de Montréal, l'Université Laval et l'Université du Québec à Montréal. Il a pour mission la promotion et la valorisation de la recherche. https://www.erudit.org/fr/ 


\section{DROBLIEMES ET SOLUTIONS}

\section{RELATIONS COMPLEXES}

Les problèmes de synonymie, au-delà d'un certain niveau de langue, présentent peut-être les difficultés les plus épineuses de la terminologie. D'une part, la synonymie, pour un même groupe d'usagers, est rarement totale, d'autre part, les distinctions qu'on fait sont en général arbitraires et peu cohérentes. Si le problème ne se posait que sur des points de haute technicité ou de raffinement d'expression, la situation ne créerait guère d'ennuis, mais souvent le problème de la synonymic se pose pour des notions de base. Il n'en faut pour preuve que la notion d'industrial relations qu'on trouve en concurrence avec labour relations et sa variante graphique labor relations, labour ou labor-management relations, employer-employee relations.

Certes, ces synonymes n'ont pas la même fréquence, ni toujours la même portée. Les deux premières expressions appartiennent presque à la langue courante, tandis qu'on trouvera les deux dernières plutôt dans des textes à prétention technique comme les conventions collectives. Mais la réalité désignée semble essentiellement la même et recouvre tous les rapports qu'un travailleur peut avoir en tant que salarié dépendant d'un employeur ${ }^{1}$. Roberts confirme d'ailleurs cette synonymie ${ }^{2}$. Mais, hors de ce sens large attesté par les dictionnaires, l'expression industrial relations tend à prendre un sens restreint, limité aux seules relations de l'entreprise avec les syndicats du personnel. C'est encore Roberts qui nous le confirme : "Others take the view that industrial relations means only unionmanagement relations" ». C'est peut-être, dans l'usage canadien, l'acception la plus courante, mais gardons-nous de donner une portée trop générale à une observation uniquement empirique. Ce qui est certain c'est que industrial relations possède un sens large et un sens étroit et qu'on le trouve fréquemment dans l'un et l'autre sens. Qu'en est-il des synonymes? Labor relations est également ambivalent, tantôt il a trait aux relations avec les syndicats, mais il peut avoir aussi

1. "Industrial relations include everything that affects the relationship of the individual worker or groups of workers to the employer ", H.S. Roberts, Dictionary of Industrial Relations, Washington, BNA Books, 1966, p. 206.

2. Id., ibid., p. 122.

3. Id., ibid., p. 206. 
le sens général comme l'atteste le Webster ${ }^{4}$. Les deux dernières expressions, à cause de leur caractère plus explicite, se prêtent moins aux mutations de sens, si bien que, lorsqu'on parle d'employer-employee relations ou de labor-management relations, on peut difficilement restreindre le contenu de ces expressions aux simples relations syndicales. C'est à union-management relations ${ }^{5}$ qu'il faudra alors songer comme équivalent de relations syndicales.

$\mathrm{Si}$ nous tournons le volet pour arriver au monde du travail francophone, comment le paysage se présente-t-il ? Comme expression générique pour désigner tous les rapports propres au monde du travail, il existe deux expressions anciennes et une nouvelle venue. Les anciennes sont RELATIONS DE TRAVAIL ${ }^{\circ}$ et RELATIONS DU TRAVAIL ${ }^{7}$. La différence entre ces deux expressions tient essentiellement à la présence de l'article. Dans la première, la suppression tend à marquer le caractère de syntagme de l'expression ; sa présence dans la seconde tend à en accentuer le caractère générique. La cloison entre les deux semble donc un peu mince pour pouvoir établir des distinctions trop tranchées. La nouvelle venue est l'expression RELATIONS INDUSTRIELLES, sans doute poussée à l'avant-scène du vocabulaire du travail par son sosie anglais. L'ouvrage de Tezenas du Montcel, publié en 1972, est l'un des premiers en France à en faire officiellement mention ${ }^{8}$. Au Canada, l'expression est courante depuis l'introduction de cette discipline parmi les matières enseignées dans les universités québécoises dans les années 40 . Le sens donné par Tezenas est très large et s'applique à l'ensemble des relations de travail et non uniquement au domaine syndical. Il n'y est pas fait mention du sens restreint qu'on trouve pourtant bien vivant au Canada.

Il convient aussi de noter un équivalent d'industrial relations mis de l'avant par l'Office de la langue française ${ }^{9}$ et consigné par le Vocabulaire de Dion ${ }^{10}$ : RELATIONS PROFESSIONNELLES. L'expression est intéressante et maniable, il ne lui manque que la caution d'un certain usage.

Au sens étroit, en plus de RELATIONS SYNDICALES, relevé plus haut, l'expression industrial relations pourrait avoir comme équivalent RELATIONS OUVRIĖRES, conformément à la loi du Québec sur la question. Mais l'expression est tombée en désuétude. Dion ne la relève même plus. Partout où l'on disait autrefois RELATIONS OUVRIERES, il parle de relations de travail ou du travail ou de relations professionnelles, ce qui semble laisser entendre que, pour lui, la distinction entre sens large et sens restreint importe peu.

4. Webster's Third New International Dictionary, Chicago, Encyclopaedia Britannica, 1966, vol. 2, p. 1259.

5. H. S. Roberts, op. cit., p. 206.

6. J. Diverrez, Politique et techniques de direction du personnel, Paris, Entreprises modernes d'édition, 1962 , p. 219.

7. D. Dugué MacCarthy, la Conduite du personnel, Paris, Dunod, $2^{*}$ éd., 1971, p. 71.

8. Henri Tezenas du Montcel, Dictionnaire des sciences de la gestion, Paris, Mame, 1972, p. 282.

9. Office de la langue française, les Organigrammes, Québec, Gouvernement du Québec, édition provisoire, 1973 , p. 161.

10. G. Dion, Vocabulaire anglais-français de relations professionnelles, Québec, Presses de l'Université Laval, 1972. 
Si l'on fait le point, nous sommes en présence en anglais d'une expression de base, industrial relations, dotée d'un sens large et d'un sens restreint. Au sens large, elle a trois synonymes, soit labor ou labour relations, employer-employee relations, labor-management relations.

Cette expression a pour correspondant de base en français RELATIONS DE ou DU TRAVAIL, avec, pour synonyme, RELATIONS PROFESSIONNELLES, proposé par l'Office de la langue française, et RELATIONS INDUSTRIELLES, consigné par Tezenas et déjà fortement enraciné au Canada.

Au sens restreint, à cause de l'ambivalence du mot labor, l'expression industrial relations peut être considérée comme synonyme de labor ou labour relations, ou même de union-management relations.

En français, comme l'expression RELATIONS OUVRIÈRES est tombée en discrédit, il ne reste comme équivalent strict d'union-management relations que l'expression RELATIONS SYNDICALES, à moins qu'on consente à faire de RELATIONS INDUSTRIELLES en français un sosie parfait d'industrial relations sur le plan sémantique. Résumons donc la question à l'aide du tableau suivant :

Expressions anglaises

1. Sens large

industrial relations

labor ou labour relations employer-employee relations labor-management relations

\section{Sens restreint}

industrial relations labor relations union-management relations
Équivalents français

$\begin{array}{ll}\text { Solutions idéales } & \text { Solutions possibles } \\ & \text { relations } \\ \text { relations professionnelles } & \begin{array}{l}\text { industrielles } \\ \text { relations de ou du } \\ \text { travail }\end{array}\end{array}$

relations

relations syndicales industrielles

relations

professionnelles

Robert Dubuc 